\title{
Ocorrência de helmintos e protozoários em hortaliças cruas comercializadas no município de Guarapuava, Paraná, Brasil
}

\author{
Helminthes and protozoa occurrence in raw vegetable from \\ commercial establishments in Guarapuava County, Paraná, Brazil
}

\author{
Leandro Mortean Ono; ; Dauton Luiz Zulpo²; Jaidson Peretti²; João Luis Garcia ${ }^{3 *}$
}

\begin{abstract}
Resumo
O consumo de hortaliças é uma das principais vias de transmissão de enteroparasitas para os seres humanos. Considerando este fato, o presente trabalho teve como objetivo avaliar a ocorrência de enteroparasitas em hortaliças comercializadas no município de Guarapuava, Paraná, Brasil. Amostras de alface lisa, alface crespa e agrião foram processadas e analisadas para verificar a presença de enteroparasitas. Das 94 amostras colhidas (55 de alface crespa, 21 de alface lisa e 18 de agrião), 42 $(44,7 \%)$ foram positivas para a presença de algum tipo de enteroparasita. As amostras de alface lisa apresentaram um risco de contaminação maior do que as de alface crespa $(\mathrm{OR}=4, \mathrm{p}<0,05)$ e as de agrião $(\mathrm{OR}=5, \mathrm{p}<0,05)$. Os principais helmintos e protozoários observados foram Ancylostomatidae $(85,7 \%)$, Strongyloides sp. (28,5\%), Giardia sp. (4,7\%) e Enterobius sp. (2,3\%). Considerando os resultados obtidos, ressalta-se a necessidade da realização de medidas preventivas para melhorar a qualidade higiênica sanitária das hortaliças, tanto pelas autoridades sanitárias como pelos consumidores.
\end{abstract}

Palavras-chave: Enteroparasitas, hortaliças, ocorrência

\begin{abstract}
Vegetables are the most important transmission source of enteroparasites to human beings. The objective of this paper was to evaluate enteroparasites occurrence in vegetables from commercial establishments of Guarapuava County, Paraná State, Brazil. Forty two samples (44.7\%) out of 94 were positive, being 15 $/ 21(71.4 \%)$ from smooth lettuce, $21 / 55$ from rough lettuce and $6 / 18(33.3 \%)$ from watercress. Smooth lettuce samples have shown a higher contamination risk than rough lettuce $(\mathrm{OR}=4, \mathrm{p}<0.05)$ and watercress $(\mathrm{OR}=5, \mathrm{p}<0.05)$. The major helminthes and protozoa observed were Ancylostomatidae $(85.7 \%)$, Strongyloides sp. (28.5\%), Giardia sp. (4.7\%) and Enterobius sp. (1.5\%). The Vegetable contaminations observed in the present work showed both necessity of government control to improve vegetable sanitary conditions, and the importance to adopt control methods before consumption by the population.
\end{abstract}

Key words: Vegetables, parasites, occurrence

\footnotetext{
1 Acadêmico dos Cursos de Ciências Biológicas e Farmácia da Universidade Estadual do Centro-Oeste - UNICENTRO. Guarapuava, PR, Brasil

2 Acadêmicos do Curso de Medicina Veterinária da Universidade Estadual do Centro-Oeste - UNICENTRO. Guarapuava, PR, Brasil.

3 Professor do Departamento de Ciências Biológicas da Universidade Estadual do Centro-Oeste - UNICENTRO. Guarapuava, PR, Brasil-e-mail: jlgarcia@unicentro.br

* Autor para correspondência.
} 


\section{Introdução}

O consumo de hortaliças é uma das principais vias de transmissão de enteroparasitas para os seres humanos, pela freqüente prática de irrigação de hortas com água contaminada por matéria fecal (TAKAYANAGUI et al., 2001), o que expõe grande parte da população às formas de transmissão de diversas enfermidades intestinais. Estes fatos constituem-se em sério problema de saúde publica, em diversos países, inclusive no Brasil, onde os coeficientes de prevalência de algumas protozoonozes ainda são, consideravelmente, elevados.

Alguns trabalhos que estudaram a contaminação de hortaliças por enteroparasitas analisaram, entre outras, amostras de alface (Lactuca sativa) e agrião (Nasturtium officinale), pelo fato de serem consumidas cruas, pela facilidade e quantidade de produção, bem como, pela possibilidade de contaminação por água e solos poluídos (MESQUITA et al., 1999; GUILHERME et al., 1999; GARCIA et al., 2004).

No Paraná, Garcia et al. (2004) avaliaram 133 amostras de hortaliças de produtores rurais do Município de Umuarama, e observaram Ascaris sp. (9,7\%), Ancilostomatideos (9,7\%), Enterobius vermicularis (1,5\%), Strongyloides sp. (0,8\%), Entamoeba sp. (3,8\%) e Giardia sp. (0,8\%). Essa contaminação foi observada em $8,4 \%$ das amostras de alface crespa, $18,2 \%$ de alface lisa, $44,4 \%$ de chicória do mato e $27,3 \%$ de chicória. Da mesma forma, Guilherme et al. (1999) encontraram altos índices de contaminação em amostras de alface lisa, alface crespa, alface mimosa, rúcula, agrião e escarola no município de Maringá.

Poucos são os trabalhos nacionais e internacionais relacionados à contaminação por enteroparasitas em hortaliças (GARCIA et al., 2004). Considerando estes fatos, o presente trabalho teve como objetivo determinar a ocorrência de helmintos e protozoários em amostras de alfaces lisa, crespa e agrião comercializadas no município de Guarapuava, Paraná, Brasil.

\section{Material e Métodos}

O presente trabalho foi desenvolvido no período de abril a dezembro de 2004, em estabelecimentos que comercializavam hortaliças (mercados e supermercados) no município de Guarapuava, que possui aproximadamente 155.161 habitantes (IBGE, 2004) e localiza-se na região centro sul do Estado do Paraná. Durante este período foram colhidas 94 amostras de hortaliças, sendo 55 de alfaces crespa, 21 de alfaces lisa, e 18 de agrião. As amostras foram obtidas nas gôndolas dos estabelecimentos e armazenadas individualmente em sacos de polietileno estéreis e prontamente encaminhadas ao Laboratório de Microbiologia da Universidade Estadual do Centro-Oeste, Departamento de Ciências Biológicas, onde foram processadas.

No Laboratório, as hortaliças foram preparadas segundo descrito anteriormente por Garcia et al. (2004), com algumas modificações. O talo era descartado junto com as folhas deterioradas. As folhas foram lavadas com auxílio de pincel em bandejas plásticas, contendo $300 \mathrm{ml}$ de solução salina com $0,005 \%$ de Extram MA $02{ }^{\circledR}$. O líquido obtido foi filtrado (gaze dobrada em quatro) e recolhido em um frasco cônico e deixado em repouso por $24 \mathrm{~h}$. A bandeja foi lavada com $50 \mathrm{ml}$ da mesma solução, recolhendo-se o líquido no mesmo frasco. Após a sedimentação, o sobrenadante foi desprezado, transferindo-se $40 \mathrm{ml}$ restantes do sedimento para tubos de ensaio cônicos. A seguir, os tubos foram centrifugados a $700 \mathrm{~g}$ por 1 minuto, desprezando-se novamente o sobrenadante. O sedimento então obtido foi ressuspenso em solução saturada de cloreto de sódio e centrifugando a $500 \mathrm{~g}$ por 1 minuto. O material flutuante foi colocado entre lâmina e lamínula e corado com solução de lugol, para posterior exame ao microscópio.

O teste de qui-quadrado foi realizado para averiguar diferenças estatísticas em relação às amostras analisadas. A probabilidade das hortaliças estarem contaminadas (OR) foi calculada pelo programa EPIINFO 6.04. Valores de $p<0,05$ foram considerados significativos. 


\section{Resultados e Discussão}

Das 94 amostras colhidas (55 de alface crespa, 21 de alface lisa e 18 de agrião), 42 (44,6\%) foram positivas para a presença de algum tipo de enteroparasita, sendo $15(35,7 \%)$ amostras positivas de alface lisa, 21 (50\%) de alface crespa e $06(14,3 \%)$ de agrião, dentre as quais, os principais enteroparasitas observados foram Ancylostomidae (85,7\%), Strongyloides sp. (28,5\%), Giardia sp. $(4,7 \%)$ e Enterobius sp. (2,3\%), conforme observado pela Tabela 1.

Tabela 1. Ocorrência de protozoários e helmintos em hortaliças comercializadas no município de Guarapuava, Paraná.

\begin{tabular}{ccccccc}
\hline Hortaliça & $\mathbf{N}^{\mathbf{1}}$ & Ancilostomídeos & Strongyloides sp. & Giardia sp. & Enterobius $\mathbf{s p .}$ & \\
\cline { 3 - 5 } Investigada & & $\mathrm{n} /(\%)^{2}$ & $\mathrm{n} /(\%)$ & $\mathrm{n} /(\%)$ & $\mathrm{n} /(\%)$ & \\
\hline Alface Crespa & 55 & $20 / 36,4$ & $4 / 7,3$ & 0 & 0 & $21 / 38,2$ \\
Alface Lisa & 21 & $12 / 57,1$ & $5 / 24,0$ & $2 / 9,5$ & 0 & $15 / 71,4$ \\
Agrião & 18 & $4 / 22,2$ & $3 / 16,7$ & 0 & $1 / 5,5$ & $6 / 33,3$ \\
\hline
\end{tabular}

${ }^{1}$ Total de hortaliças investigadas

${ }^{2} \mathrm{n}=$ número de amostras detectadas como positivas

${ }^{3}$ Total de hortaliças positivas

Resultados similares foram observados por Takayanagui et al. (2001), Guilherme et al. (1999) e Simões et al. (2001), com ocorrências de 33\%, 16,6\% e 14,5\% respectivamente, considerando que nos três trabalhos citados outras variedades de hortaliças foram acrescentadas às análises. Mota et al. (1983) também verificaram altos níveis de enteroparasitas em hortaliças consumidas cruas na cidade de Curitiba. Além destes, Garcia et al. (2004) verificaram a ocorrência de helmintos em 17,3\% e de protozoários em $4,5 \%$ das hortaliças produzidas no município de Umuarama.

Oliveira e Germano (1992) afirmaram que as diferenças entre os níveis de contaminação nas variedades de hortaliças parecem estar associadas, fundamentalmente, com as condições sanitárias do ambiente em que são cultivadas. Os vegetais que crescem em solos poluídos podem carrear ovos de helmintos parasitas de humanos, como Ascaris sp. e Trichuris sp., que são mais resistentes às condições externas e que não requerem hospedeiros intermediários (GUILHERME et al., 1999).

A probabilidade (OR) dos diferentes tipos de hortaliças estarem contaminados por parasitas foi analisada. Houve uma diferença estatística significativa quando foram comparados as amostras de alface lisa com as amostras de alface crespa e agrião $(p=0,02)$. As amostras de alface lisa apresentaram um risco maior de contaminação por enteroparasitas do que as amostras de alface crespa e agrião, com $\mathrm{OR}=4(\mathrm{p}=0,009)$ e $\mathrm{OR}=5(\mathrm{p}=$ $0,01)$, respectivamente. Estes dados foram de certa forma conflitantes, pois se esperava uma contaminação maior da alface crespa, pela sua capacidade de reter sujidades, bem como do agrião, pelas suas características de cultivo.

A contaminação de hortaliças pode ocorrer na horta, resultante da utilização de água de irrigação ou adubos inadequados, no transporte ou nos pontos de venda e também pelas sucessivas manipulações (TAKAYANAGUI et al., 2001)

As altas contaminações por enteroparasitas observadas no presente estudo ressaltam a importância das medidas de prevenção sobre as hortaliças, que devem ser adotadas pelas autoridades sanitárias, produtores e pelos consumidores, durante a produção, comercialização e antes do consumo. 


\section{Referências}

GARCIA, J.L.; JAHN, T.R.; FERMO, E.É.; NEVES, U.S.; PURETZ, E. Evaluation of helminthes and protozoa in raw vegetables produced in Umuarama, Paraná State. Arquivos de Ciências Veterinárias e Zoologia da Unipar, Umuarama, v.7, p. 7-10, 2004.

GUILHERME, A.L.F.; ARAUJO, S.M.; FALAVIGNA, D.L.M.; PUPULIM, A.R.T.; DIAS, M.L.G.; OLIVEIRA, H.S.; MAROCO, E.; FUKUSHIGUE, Y. Prevalência de enteroparasitas em horticultores e hortaliças da Feira do Produtor de Maringá, Paraná. Revista da Sociedade Brasileira de Medicina Tropical, Rio de Janeiro, v.32, n.4, p.405-411. 1999.

IBGE. Resultados do censo 2000. Disponível em: $<$ http:// www.ibge.gov.br/cidadesat/default.php.htm $>$ Acesso em: 31 out. 2004.

MESQUITA, V.C.L.; SERRA, C.M.B.; BASTOS, O.M.P. UCHOA, C.M.A. Contaminação por enteroparasitas em hortaliças comercializadas nas cidades de Niterói e Rio de Janeiro, Brasil. Revista da Sociedade Brasileira de Medicina Tropical, Rio de Janeiro, v.32, p.363-366, 1999.

MOTA, C.C.S.; ELIAS, A.; MIKOSZEWSKA, I.; VIEIRA, H.R.A.; PICHET NETO, J.; VASQUES, R.M.R.; ALMEIDA, A.A.; GAISSLER, M.S.; BEATRIZ, R.; MOTA, R.M.T.C.S. Condições higiênico-santárias de hortaliças comercializadas em Curitiba_PR. (BRASIL). In: CONGRESSOBRASILEIRODECIÊNNCIAETECNOLOGIA DE ALIMENTOS, 16., 1983, Brasília. Resumos... Brasília: CBCTA, 1983.p.125.
OLIVEIRA, C.A.F.; GERMANO, P.M.L. Estudo da ocorrência de enteroparasitas em hortaliças comercializadas na região metropolitana de São Paulo, SP, Brasil: I - Pesquisa de helmintos. Revista de Saúde Pública, São Paulo, v.26, p.283-289, 1992.

SIMÕES, M.; PISANI, B.; MARQUES, E.G.L.M.; PRANDI, M.A.G.P.; MARTINI, M.H.M.; CHIARINI, P.F.T.; ANTUNES, J.L.F.; NOGUEIRA, A.P.N. Hygienic sanitary conditions of vegetables and irrigation water from kitchen garden in the municipality of Campinas, SP. Brazilian Journal of Microbiology, São Paulo, v.32, p.331-333, 2001.

TAKAYANAGUI, O.M.; OLIVEIRA, C. D.; BERGAMINI, A.M.M.; CAPUANO, D.M.; OKINO, M.H.T.; FEBRÔNIO, L.H.P.; SILVA, A.A.M.C.; OLIVEIRA, M.A.; RIBEIRO, E.G.A.; TAKAYANAGUI, A.M.M. Fiscalização de verduras comercializadas no município de Ribeirão Preto, SP. Revista da Sociedade Brasileira de Medicina Tropical, Rio de Janeiro, v.34, n.1, p.37-41, 2001. 